\title{
Structural Characterization of Sialic Acid Synthase by Electrospray Mass Spectrometry-a Tetrameric Enzyme Composed of Dimeric Dimers
}

\author{
Hsin-Hung Huang, ${ }^{*}$ Hsin-Kai Liao, and Yu-Ju Chen* \\ Institute of Chemistry, Academia Sinica, Taipei, Taiwan \\ Tzann-Shun Hwang, ${ }^{+}$Yi-Han Lin, and Chun-Hung Lin ${ }^{\dagger}$ \\ Institute of Biological Chemistry, Academia Sinica, Taipei, Taiwan
}

Sialic acid synthase (NeuB) encoded by the neuB gene catalyzes the condensation of $\mathrm{N}$-acetylmannosamine and phospho(enol)pyruvate to form $\mathrm{N}$-acetylneuraminic acid. The enzyme is essential for the biosynthesis of polysialic acid, a capsular sugar polymer functioning as a virulent factor and antiphagocytic barrier. This report demonstrates the first characterization on the quaternary structure of NeuB from Escherichia coli (EcNeuB) and Streptococcus agalactiae (SaNeuB) by nanoflow electrospray ionization mass spectrometry (ESI-MS). Under non-denaturing conditions, Tris buffer was observed to induce a higher ratio of tetramer/dimer of NeuB in the ESI mass spectra, providing supportive evidence for the existence of a "structurally-specific" tetramer. The instrument parameters were found to significantly affect the ratio of detected tetramer/dimer in ESI mass spectra. The harshest conditions, including high desolvation voltages and pressure in the collision cell, led to enhanced detection of the $160 \mathrm{kDa}$ tetramer. The prevalence of dimeric form is likely the cause in loss of tetramer stability in gas-phase arising from insufficient collisional cooling, which implies an asymmetric assembly, possibly composed of dimeric dimers. Most interestingly, the hypothesis was further supported by chemical cross-linking of SaNeuB, in which the reaction of shorter linker yielded mainly the dimer whereas that of longer linker produced both dimer and tetramer. Furthermore, the ESI-MS analysis can reflect dramatic change of $\mathrm{pH}$-dependent quaternary structure in association with enzyme activity, suggesting the tetrameric form may be the primary species responsible for the enzyme catalysis. (J Am Soc Mass Spectrom 2005, 16, 324-332) (C 2004 American Society for Mass Spectrometry

$\mathrm{N}$ oncovalent interactions of proteins contribute to the structural basis of many biological processes. Proteins interact with other biomolecules to form supermolecular assembly via hydrophobic, electrostatic, hydrogen bonding and van der Waals interactions [1]. The protein quaternary structure plays an important role in close association with the activity and physiological function. Many enzymes in their active forms exist as oligomers, most commonly as dimers or tetramers [2]. When a protein is composed of multiple subunits such as tetramer, different interactions in the subunit interface are usually relevant to the integrity of quaternary structure. Therefore, to obtain such information certainly provides the key to address

Published online January 14, 2005

Address reprint requests to Dr. Y.-J. Chen, Institute of Chemistry, Academia Sinica, 128 Academia Road, Section 2, Nan-kang, Taipei 11529, Taiwan. E-mail: yjchen@chem.sinica.edu.tw

* Also at the National Central University, Taoyuan, Taiwan.

† Also at the Institute of Biochemical Sciences, National Taiwan University, Taipei, Taiwan. various issues, including catalysis and biological function.

On account of the intrinsic nature of accurate measurement of molecular masses, mass spectrometry has become an indispensable tool to study noncovalent interactions for protein complexes or oligomers. The detection of noncovalent complexes in solution is of great interest owing to available information about the stoichiometry and/or relative binding affinity of complexes [3-5]. Many examples have successfully demonstrated that the structural specificity in electrospray ionization mass spectrometry (ESI-MS) is consistent with those obtained by solution-phase spectroscopic techniques [6-8]. The unique information provides insight for understanding related biological function [9-13]. An increasing number of evidences suggest that the ESI-MS observations of the weakly bound protein assembly reflect, to some extent, the nature of the interaction in the condensed phase [13-15]. Dissociation of gas-phase complexes may provide information about their solution-phase conformations. As pointed out by 

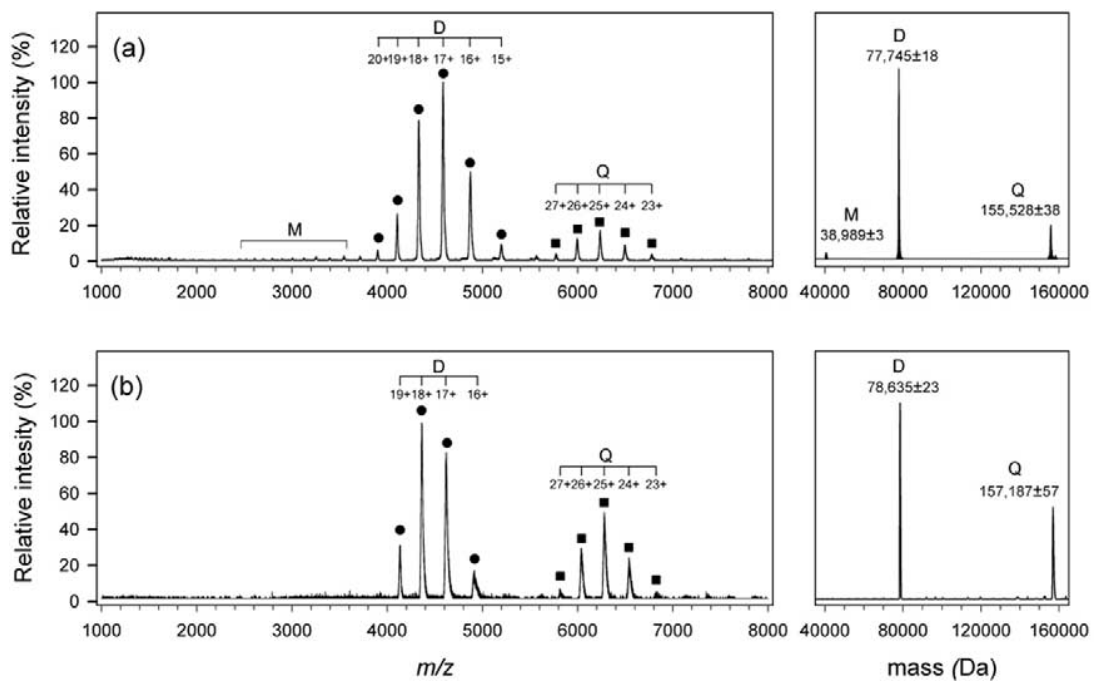

Figure 1. ESI-MS spectra of SaNeuB and the deconvoluted mass spectra under nondenaturing conditions in (a) ammonium acetate $10 \mathrm{mM}, \mathrm{pH} 8.0$; (b) Tris $0.1 \mathrm{mM}, \mathrm{pH}$ 8.0. Q: tetramer; D: dimer; M: monomer. The accelerating/declustering potential DP and DP2 were set to 100 and $80 \mathrm{~V}$, respectively. The pressure in the collision cell was $7.1 \times 10^{-3}$ torr.

Robinson and coworkers, the precise determination of protein oligomeric state critically depends on the interactions between associating subunits [16]. Electrostatic interaction is believed to be enhanced whereas most of the hydrophobic interactions are disrupted in the gas phase. For selected systems, it becomes convincingly evident that the structural integrity of several multimeric proteins, already well characterized by X-ray crystallography, can be reflected in the native ESI-MS measurement [16-18]. Recently, ESI-MS has been applied to probe the architecture of the multiprotein complexes $[19,20]$, and the results shed light on the potential of employing mass spectrometry to unravel the integrity and interaction of associating subunits.

Pioneering work utilizing ESI-MS to study noncovalent complex had optimized the solution condition and ESI interface parameters to successfully transfer the structural-specific macromolecular assembly from solution to gas-phase [14]. To preserve the fragile noncovalent protein complex, it is generally accepted that "gentle" conditions should be maintained by mild desolvation voltages and low temperature in the atmosphere-vacuum ESI interface conditions [21]. However, the detection of very large multi-protein complex represents extreme cases where high interface pressure and accelerating voltages are usually preferred [22]. Due to the mass-dependent ion transmission in the interface region and quadrupoles, the analysis of large multiprotein or multi-subunit assembly by ESI-MS still represents an analytical challenge which is far from routine operation. It becomes a prerequisite to fine tune sample preparation and ESI interface conditions. Most of the noncovalent protein complexes studied by ESI-MS to date are structurally well-known examples. To validate if the mass spectra can reflect the solution phase structure, it is necessary to correlate the specificity of the observed oligomeric forms with that in the solutionphase. Special attention has to be given to experimental and instrumental parameters that may affect the solution properties (e.g., $\mathrm{pH}$ and ionic strength) and gas phase properties (e.g., declustering potential and pressure) during ionization and gas-phase transfer.

In this study, we characterize the multimeric form of sialic acid synthase $(39 \mathrm{kDa})$ encoded by the neuB gene using nanoflow electrospray quadrupole time-of-flight (Q-TOF) mass spectrometer under nondenaturing conditions. Both enzymes from Streptococcus agalactiae (abbreviated as SaNeuB) and E. coli (abbreviated as Ec$\mathrm{NeuB}$ ) are investigated. In several pathogenic bacteria, including E. coli K1, Neisseria meningitides, and group B Streptococcus, the enzyme is involved in the biosynthesis of polysialic acids that are capsular polysaccharides to provide the bacteria with an antiphagocytic barrier and acts as essential virulence determinants in the evasion of host defense [23, 24]. Sialic acid synthase catalyzes the condensation reaction of $\mathrm{N}$-acetylmannosamine with phospho(enol)pyruvate (PEP) to give N-acetylneuraminic acid [25]. There are controversial reports on the oligomeric state of SaNeuB and EcNeuB using different techniques [26, 27]. Therefore, we are interested in determination of the oligomerization states of NeuB by nanoflow ESI-MS, especially the active component responsible for enzyme activity. With the concurrence of tetrameric and dimeric SaNeuB in mass spectra under various conditions (Figure 1), it is intriguing to study whether the dimer is "structurally-specific" in solution or arise from gas-phase dissociation of tetramer. In addition to studying the native noncovalent oligomers, chemical cross-linking experiments were conducted to obtain information on the geometry of the NeuB. The ultimate goal is to decipher the assembly of the quaternary structure and the structure- 
function relationship associated with the enzyme catalysis.

\section{Experimental}

\section{Protein Expression and Purification}

The proteins were expressed in the E. coli and purified according to the previous procedure [27]. After the purification by a HiTrap chelatings High Performance (HP) and HiTrap Q-sepharose HP column, the desired proteins were dialyzed in Tris buffer or ammonium acetate $(\mathrm{pH} 8.0)$ to give $>97 \%$ homogeneity. On the basis of thiobarbituric assay [28], SaNeuB and EcNeuB were usually obtained with activity of $40 \mu \mathrm{mole} / \mathrm{mg}$ min.

\section{Cross-Linking Reaction of SaNeuB}

The cross-linking reactions were performed according to the reported method of Davis and Stark, and Chiou et al. $[29,30]^{\circ}$ using $^{\circ}$ dimethyl $^{\circ}$ adipimidate $^{\circ}(\mathrm{DMA})^{\circ}$ and dimethyl suberimidate (DMS). DMA and DMS were purchased from Pierce Chemical (Rockford, IL). The protein $(\mathrm{SaNeuB})$ and cross-linking agents were dissolved separately in $0.2 \mathrm{M}$ triethanolamine- $\mathrm{HCl}(\mathrm{pH}$ $8.0)$ to give the protein of $0.2-6.0 \mathrm{mg} / \mathrm{ml}$ and crosslinker of $0.5-10 \mathrm{mg} / \mathrm{ml}$, respectively. The two solutions were mixed together immediately after they had been prepared. The reaction was carried out at $25^{\circ} \mathrm{C}$ for $1 \mathrm{~h}$, stopped by adding $1 \%$ SDS and $0.1 \%$ 2-mercaptoethanol (wt/vol), and followed by heating at $100{ }^{\circ} \mathrm{C}$. Reaction products were examined by SDS-PAGE analysis.

\section{Electrospray Ionization Mass Spectrometry}

Prior to mass analysis, the samples were desalted by microcon centrifugal filter (cut-off $=10,000 \mathrm{Da}$, Millipore, Billerica, MA) in Tris buffer or ammonium acetate (pH 8.0). Six dilution/concentration steps were performed at $4{ }^{\circ} \mathrm{C}$ and $12,000 \mathrm{~g}$. The sample solution was diluted to a final tetramer concentration of $10 \mathrm{pmol} / \mu \mathrm{L}$. ESI mass spectra of NeuB were obtained in the positive ion mode using a quadrupole time-of-flight mass spectrometer (QSTAR Pulsar i, Applied Biosystem/MDS Sciex, Foster City, CA) equipped with a nanospray ionization source. Typically, $2 \mu \mathrm{L}$ desalted sample solution of $10 \mu \mathrm{M}$ tetramer concentration was nanosprayed from a nanospray tip (Protana, Odense, Denmark). For non-denaturing ESI mass analysis, the nanospray ionization was performed using ammonium acetate or Tris (0.1-10 mM, $\mathrm{pH} 4-11)$ to maintain native conformation of NeuB. The ionization voltage is typically ${ }^{\circ}$ increased ${ }^{\circ}$ slowly ${ }^{\circ}$ from ${ }^{\circ} 700^{\circ}$ to ${ }^{\circ} 1200^{\circ} \mathrm{V}^{\circ}$ until ${ }^{\circ}$ the ${ }^{\circ}$ ion current was stable. The mass scan range was set between $\mathrm{m} / \mathrm{z} 1000$ and 10,000. To optimize the detection sensitivity and preserve the integrity of noncovalently bound NeuB assembly, the pressure in collision cell was varied from $1.6 \times 10^{-3}$ to $7.1 \times 10^{-3}$ torr. The acceler- ation/desolvation voltages, DP and DP2, ranged from 5 to $100 \mathrm{~V}$ and 100 to $280 \mathrm{~V}$, respectively. Calibration of the mass spectra was performed by using myoglobin ( 5 $\mu \mathrm{M})$. A typical mass spectrum was acquired by the sum of 100 scans, smoothed using a Gaussian filter. The molecular mass of different multimeric state in each mass spectrum was obtained by deconvolution using Analyst QS (Applied Biosystems) software.

\section{Results and Discussions}

\section{Determination of the Quaternary Structure of SaNeuB by Nanoflow ESI-MS}

In this study, all the samples were desalted by ultrafiltration centrifugal filter (MW cut-off $=10,000 \mathrm{Da}$ ) in different buffers including ammonium acetate, ammonium ${ }^{\circ}$ bicarbonate, ${ }^{\circ} \mathrm{HEPES}^{\circ}$ and $^{\circ}$ Tris $^{\circ}$ buffer. $^{\circ}$ The $^{\circ}$ oligomeric state of $\mathrm{SaNeuB}$ was studied using nanoflow electrospray ionization Q-TOF MS under non-denaturing ${ }^{\circ}$ conditions ${ }^{\circ}$ optimized 9 for ${ }^{9}$ the tetramer. Figure $1 a^{\circ}$ and b shows that two ion series from $\mathrm{m} / \mathrm{z} 1000$ to 10,000 were observed ${ }^{\circ}{ }^{\circ}{ }^{\circ}$ ammonium ${ }^{\circ}$ acetate ${ }^{\circ}$ and ${ }^{\circ}$ Tris buffer, ${ }^{\circ}$ respectively, ${ }^{\circ}$ as $^{\circ}$ well $^{\circ}$ as $^{\circ}$ their ${ }^{\circ}$ corresponding $^{\circ}$ masses ${ }^{\circ}$ deduced by Analyst QS deconvolution. When the ESI-MS was performed $^{\circ}$ using $^{\circ}$ ammonium $^{\circ}$ acetate $^{\circ}\left(\right.$ Figure $\left.^{\circ} 1 \mathrm{a}\right){ }^{\circ}$ the peaks observed in the range of $5700-7000 \mathrm{~m} / \mathrm{z}$ with the charge ${ }^{\circ}$ states $^{\circ}$ ranging $^{\circ}$ from ${ }^{\circ} 27+{ }^{\circ}$ to $^{\circ} 23+{ }^{\circ}$ correspond $^{\circ}$ to deconvoluted molecular mass of $155,528 \pm 38 \mathrm{Da}$, in association with the molecular mass of tetrameric SaNeuB (38,836 Da calculated from the primary sequence of the monomeric SaNeuB). Multiply charged dimer ions were observed predominantly. The dominant set of peaks with the charge states from $20+$ to $15+(\mathrm{m} / \mathrm{z}$ from 3900 to 5200 ) were identified with a mass of $77,745 \pm 18$ $\mathrm{Da}$, in agreement with the value of dimeric NeuB. The detection of tetramer was greatly enhanced by the non-denaturing ESI-MS analysis in Tris buffer, as shown $^{\circ}$ in $^{\circ}$ Figure $^{\circ} 1 \mathrm{~b}^{\circ}{ }^{\circ}$ Compared $^{\circ}$ to ${ }^{\circ}$ the ${ }^{\circ}$ deconvoluted masses of dimer and tetramer obtained in ammonium acetate, ${ }^{\circ}$ Figure ${ }^{\circ} 1 b^{\circ}$ shows ${ }^{\circ}$ significant ${ }^{\circ}$ increases ${ }^{\circ}$ of ${ }^{\circ} 890^{\circ} \mathrm{Da}$ and $1559 \mathrm{Da}$ in the dimeric $(78,635 \pm 23 \mathrm{Da})$ and tetrameric $(157,187 \pm 57 \mathrm{Da})$ peaks of SaNeuB in Tris buffer, respectively. The observed masses of both dimer

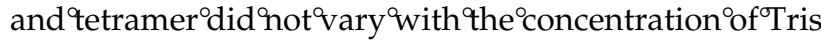
buffer $(0.1-5 \mathrm{mM})$. The observation indicates that the Tris molecules may bind to the protein and increased intensity of tetrameric peak in mass spectra, suggesting the contribution of Tris to the stabilization of the tetrameric structure. The absence of monomer, trimer, pentamer or other higher-order oligomers reveals that the dimeric and tetrameric forms reflect the structurally-specific association of sialic acid synthase in solution. The possibility of nonspecific aggregation from dimer to tetramer can be excluded because the ratio of dimer/tetramer does not change with the protein concentration (data not shown). The dimer and tetramer exhibit narrow charge state distribution (five charge states), which is relevant to the retention of the compact 

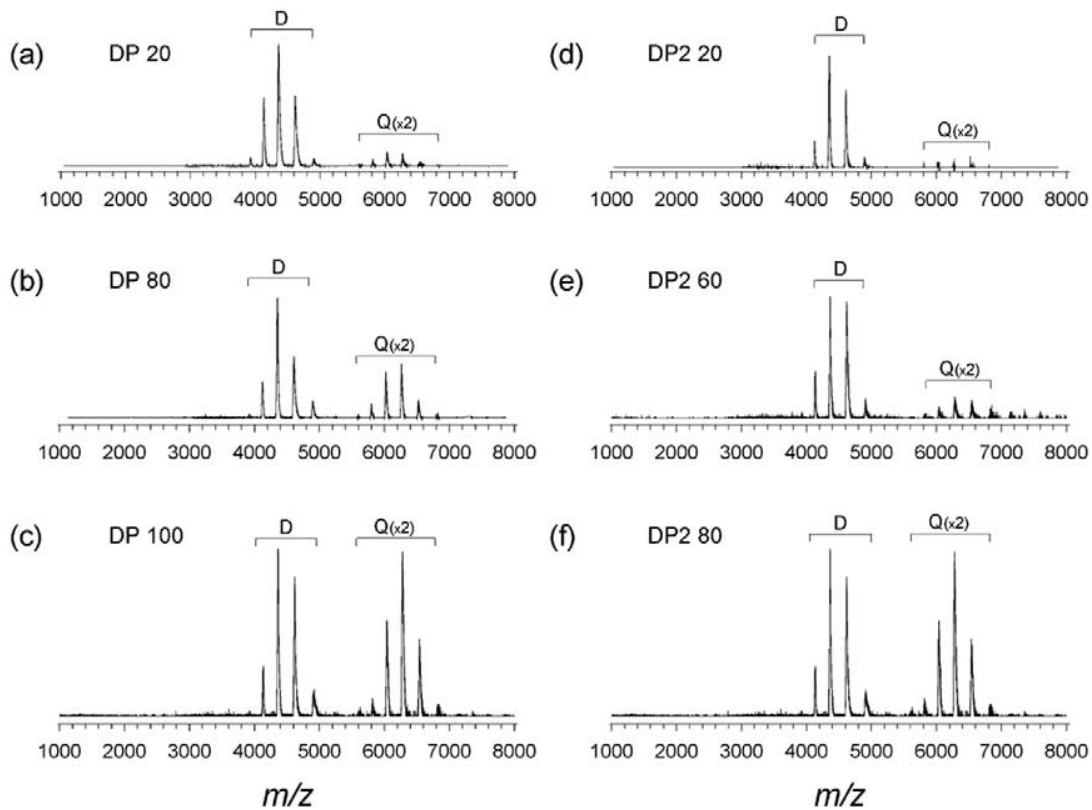

Figure 2. Effect of the declustering potentials, DP and DP2, in the ESI interface on the detection of tetramer of SaNeuB in $0.1 \mathrm{mM}$ Tris $(\mathrm{pH} 8.0)$ and CAD gas pressure in $7.1 \times 10^{-3}$ torr. (a) $\mathrm{DP}=20$, DP2 $=80 ;(\mathbf{b}) \mathrm{DP}=80, \mathrm{DP} 2=80 ;(\mathbf{c}) \mathrm{DP}=100, \mathrm{DP} 2=80 ;(\mathbf{d}) \mathrm{DP}=100, \mathrm{DP} 2=20 ;(\mathbf{e}) \mathrm{DP}=100, \mathrm{DP} 2=$ 60; (f) DP $=100, \mathrm{DP} 2=80$. The applicable voltages for DP and DP2 are 5-100 and 15-80 V, respectively. The pressure in the collision cell during data acquisition is $7.1 \times 10^{-3}$ torr. Q: tetramer; D: dimer.

structure ${ }^{\circ}{ }^{\circ}{ }^{\circ}$ the ${ }^{\circ}$ gas $^{\circ}$ phase $^{\circ}[14]$. $^{\circ}$ The $^{\circ}$ result $^{\circ}$ supports $^{\circ}$ the preservation for the native state of the observed SaNeuB. It is interesting to note that the Tris buffer yields relatively narrower multiple charge distribution of dimer and tetramer than ammonium acetate, which may suggest its superior capability to preserve the native structure.

\section{Influence of Instrument Parameters on the Detection of Noncovalent NeuB Complex}

Declustering potential. To enhance the detection sensitivity while preserving the integrity of the noncovalent protein assembly, the acceleration voltage in the ESI interface was first optimized. There are two important ion optics in the interface region which can be adjusted for the extent of ion transmission and desolvation, including the orifice-skimmer voltage (declustering potential, DP), and skimmer-first quadrupole voltage (declustering ${ }^{\circ}$ potential $\left.{ }^{\circ},{ }^{\circ} \mathrm{DP} 2\right) .{ }^{\circ}{ }^{\circ}$ Figure $2{ }^{\circ}$ presents ${ }^{\circ} \mathrm{ESI}^{\circ} \mathrm{mass}$ spectra of SaNeuB sprayed from the Tris buffer solution of $\mathrm{pH} 8.0$ at different accelerating voltages. Increasing the accelerating voltage DP led to the higher relative abundance ${ }^{\circ}$ of $^{\circ}$ tetramer ${ }^{\circ}\left(\right.$ Figure $\left.^{\circ} 2 \mathrm{a},{ }^{\circ} \mathrm{b},{ }^{\circ} \mathrm{C}\right) .{ }^{\circ}{ }^{\circ}$ The ${ }^{\circ}$ optimum detection of tetramer was found at maximum applicable $^{\circ}$ voltage ${ }^{\circ}$ of $^{\circ} 100^{\circ} \mathrm{V}^{\circ}$ (Figure $\left.{ }^{\circ} 2 \mathrm{c}\right)$. $^{\circ}$ Likewise, ${ }^{\circ}$ the ${ }^{\circ}$ increase of DP2 exhibited similar effect on the ratio of tetramer/ $\operatorname{dimer}^{\circ}$ (Figure ${ }^{\circ} 2 \mathrm{~d},{ }^{\circ}$ e, ${ }^{\circ}$ f). ${ }^{\circ}$ Generally ${ }^{\circ}$ speaking, ${ }^{\circ}$ higher accelerating voltage offers more initial kinetic energy required for transmission of higher $\mathrm{m} / \mathrm{z}$ ions. Efficient desolvation of larger $\mathrm{m} / \mathrm{z}$ ions is also facilitated at higher acceleration voltage. However, increased acceleration voltages often generate more energetic collisions with residual gas in the interface, leading to possible insource dissociation. Thus, compromise usually has to be made to either preserve noncovalent complexes or alternatively cause the disassembly of individual subunits by the gas-phase collisional-induced dissociation. In general, the weakly-bound noncovalent protein assembly preferentially exists under extremely gentle condition $^{\circ}\left[14,{ }^{\circ} 21\right] . .^{\circ}$ Our $^{\circ}$ results $^{\circ}$ present $^{\circ}$ an ${ }^{\circ}$ interesting case that highest applicable accelerating voltages result in the maximum abundance of tetramer, in contrast to most of the reports in which higher accelerating voltages in the ESI interface activate the dissociation of the fragile noncovalent complex. In general, they are often reduced to maintain the intact structure. Until recently, high desolvation voltages were found to be required for transmission ${ }^{\circ}$ of $^{\circ}$ very $^{\circ}{ }^{\circ}$ arge ${ }^{\circ}$ supermolecules $\left.{ }^{\circ}\left[22,{ }^{\circ} 31\right]\right]^{\circ}$, in agreement with our observed tendency for detection of $160 \mathrm{kDa}$ tetrameric NeuB.

Pressure effect. Another crucial factor is the pressure in the ${ }^{\circ}$ interface ${ }^{\circ}$ and $^{\circ}$ collision $^{\circ}{ }^{\circ}$ cell..$^{\circ}$ Figure ${ }^{\circ} 3^{\circ}$ illustrates ${ }^{\circ}$ the effect ${ }^{\circ}$ of $^{\circ}$ the ${ }^{\circ}$ collision ${ }^{\circ}$ cell ${ }^{\circ}$ pressure ${ }^{\circ}$ ranging ${ }^{\circ}$ from ${ }^{\circ} 1.6^{\circ}$ to $7.1 \times 10^{-3}$ torr on the SaNeuB mass spectra obtained at $\mathrm{pH}$ 8.0. At the pressure of $1.6 \times 10^{-3}$ torr, the major species corresponds to the dimeric form of SaNeuB (Figure $\left.^{\circ} 3 a\right)^{\circ}$ with $^{\circ}$ trace $^{\circ}$ amount $^{\circ}$ of $^{\circ}$ tetramer $^{\circ}$ in $^{\circ}$ mass spectra..$^{\circ}$ Obviously, $^{\circ}$ the ${ }^{\circ}$ bimodal $^{\circ}$ distribution $^{\circ}$ of ${ }^{\circ}$ the spectra was significantly influenced by the pressure in the collision cell. Although both the dimer and tetramer 
(a)

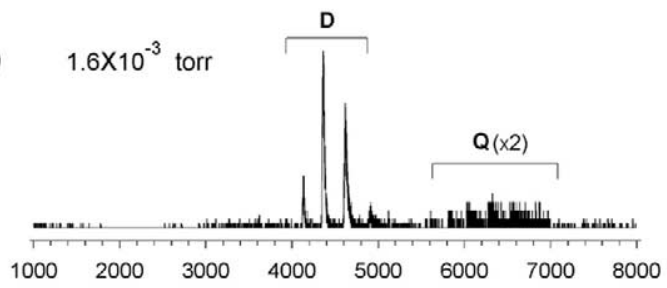

(b)

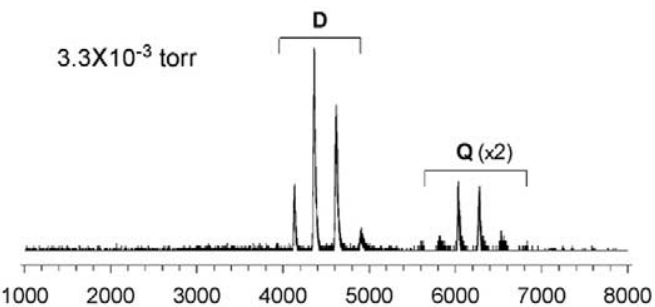

(c)

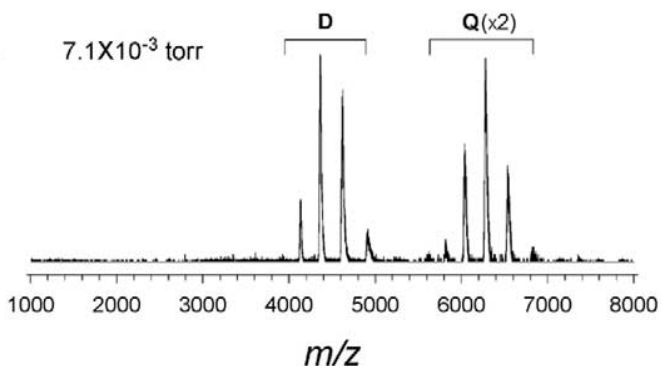

Figure 3. Effect of the pressure in the collision cell on the detection of tetramer of SaNeuB in $0.1 \mathrm{mM}$ Tris $(\mathrm{pH} 8.0)$ and $\mathrm{DP} / \mathrm{DP} 2$ is $100 / 80$. (a) $1.6 \times 10^{-3}$ torr; (b) $3.3 \times 10^{-3}$ torr; (c) 7.1 $\times 10^{-3}$ torr. The applied voltages for DP and DP2 are 100 and 80 $\mathrm{V}$, respectively. Q: tetramer; D: dimer.

intensities increase with elevating collision cell pressure, the intensity of tetramer increases more considerably. Preservation of the tetramer is most facilitated at the highest applicable pressure of $7.1 \times 10^{-3}$ torr (Figure ${ }^{\circ}$ c). ${ }^{\circ}$ The ${ }^{\circ}$ same ${ }^{\circ}$ phenomenon has been attributed to the pressure effect in $^{\circ}$ the $^{\circ} \mathrm{ESI}^{\circ}$ interface ${ }^{\circ}$ that ${ }^{\circ}$ the pressure $^{\circ}$ in $^{\circ}$ the $e^{\circ}$ first pumping stage of the ESI has significant influence on the ${ }^{\circ}$ ion ${ }^{\circ}$ abundance ${ }^{\circ}$ of $^{\circ}$ a noncovalent ${ }^{\circ}$ complex $^{\circ}\left[32,{ }^{\circ} 33\right] .{ }^{\circ}$ Elevating ${ }^{\circ}$ the ${ }^{\circ}$ pressure in ${ }^{\circ}$ the source ${ }^{\circ}$ region ${ }^{\circ}$ can $^{\circ}$ greatly $^{\circ}$ enhance ${ }^{\circ}$ the ${ }^{\circ}$ detection ${ }^{\circ}$ efficiency of large ${ }^{\circ}$ noncovalent ${ }^{\circ}$ protein $^{\circ}$ complexes. $^{\circ}$ Tahallah ${ }^{\circ} \mathrm{t}^{\circ} \mathrm{al} .{ }^{\circ}$ and ${ }^{\circ} \mathrm{Krutchin} s k y^{\circ} \mathrm{e} \mathrm{t}^{\circ} \mathrm{al} .{ }^{\circ}\left[33,{ }^{\circ}, 3\right]^{\circ}$ pointed $^{\circ}$ out that the transverse and axial ion velocity components of the energetically excited ions are significantly reduced by the ${ }^{\circ}$ collisions $^{\circ}$ with $^{\circ}$ neutral ${ }^{\circ}$ molecules ${ }^{\circ}$ and ${ }^{\circ}$ thus ${ }^{\circ}$ result ${ }^{\circ}$ in better transmission into the TOF analyzer. The increased pressure contributes to a shorter distance between two successive collisions so that more frequent but less energetic, less destructive collisions are generated to enhance collisional cooling of the protein assembly. For a noncovalent protein complex with molecular mass greater than several kilodaltons, more collisional cooling is essential to reduce the high kinetic energy acquired in the ESI source. In addition to the abovementioned collisions in the interface, it is surprising that the pressure in the collision cell also plays $\mathrm{a}^{\circ}$ role $^{\circ} \mathrm{in}^{\circ}$ collisional ${ }^{\circ}$ cooling. The ${ }^{\circ}$ profound $^{\circ}$ effect $^{\circ}$ as $^{\circ}$ shown in Figure ${ }^{\circ} 3^{\circ}$ indicates that the $160 \mathrm{kDa}$ tetramer still possesses certain kinetic energy after passing through the high pressure regions of ESI interface (2.5 torr), first and second RF-quadrupoles $\left(10^{-2}\right.$ torr). Obviously, the extra internal energy of the tetramer is unable to be completely cooled down by collisions in the above regions, yet ${ }^{\circ}$ presumably ${ }^{\circ}$ sufficient ${ }^{\circ}$ to ${ }^{\circ} e^{9}$ transferred to the vibrational mode, and subunit-subunit dissociation is therefore induced.

\section{The Tetramer Can be Related to the Enzyme Activity}

Although mass spectrometry is a valuable tool for the structural characterization of protein complex, it is more important to identify which oligomeric component is related to the enzyme activity. Thus, the structural specificity of the oligomeric states observed in ESI-MS has to be validated. The ${ }^{\circ}$ activity ${ }^{\circ}{ }^{\circ}{ }^{\circ}{ }{ }^{\circ} \mathrm{NeuB}^{\circ}$ was found ${ }^{\circ}$ to ${ }^{\circ} \mathrm{be}^{\circ} \mathrm{pH}^{\circ}$ dependent. ${ }^{\circ}$ Figure $^{\circ} 4^{\circ}$ shows ${ }^{\circ}$ the ESI-MS spectra of SaNeuB at pH 4.0-11.0 in 0.1 mM Tris buffer. The distribution of monomer, dimer and tetramer was sensitive to the $\mathrm{pH}$ variation, particularly the tetramer. The tetrameric species $(28+$ to $23+, \sim m / z 6000)$ was only evident throughout relatively neutral $\mathrm{pH}$ (7.5-8.5). Lower or higher $\mathrm{pH}$ not only resulted in a dramatic change in the ESI-MS spectra measured under identical conditions, but also diminished the tetramer in ESI-MS. The formation of the tetramer in ESI-MS at pH 7.5-8.5 further confirms the observed oligomeric state arising from specific associations in solution instead of random aggregation during electrospray process. At $\mathrm{pH} 4.0$, SaNeuB dissociated to give the monomer while dimer still remained as the dominant species. At $\mathrm{pH}$ 11.0, the dominant dimer shifted to two charge-state distributions, indicative of two conformations of monomeric SaNeuB over the range of $\mathrm{m} / \mathrm{z} 1000$ to 3000 . In addition to the aforementioned monomer at lower $\mathrm{m} / \mathrm{z}(<2000$ $\mathrm{m} / \mathrm{z}$, designated as $\mathbf{M})$, another series of intense peaks appear ${ }^{\circ}$ at $^{\circ}$ significantly ${ }^{\circ}$ higher $\mathrm{m} / \mathrm{z}(2000-3000 \mathrm{~m} / \mathrm{z}$ designated as $\mathrm{M}^{\prime}$ ) corresponding to the lower charge states $(27+$ to $13+)$. The bimodal distribution of monomeric SaNeuB suggested different mechanisms of ion formation. The lower charge states $\left(\mathrm{M}^{\prime}\right)$ implied that the dissociation occurs at later stage of ESI interface after the charge states of the oligomeric states have ${ }^{\circ}$ been determined ${ }^{\circ}[21]^{\circ}$ Because the change was accompanied by the simultaneous decrease of the dimer/tetramer abundance, it is likely that the basic $\mathrm{pH}$ completely destabilized the native SaNeuB and caused dissociation to yield a more compact monomer $\left(\mathrm{M}^{\prime}\right)$ in the gas phase.

The activity assay of SaNeuB indicates a narrow activity range between $\mathrm{pH} 7.0-9.5$ (retaining $>40 \%$ of the full activity) with an optimum at $\mathrm{pH}$ 8.0. The activity of SaNeuB in Tris buffer is significantly higher than that in ammonium acetate. The $\mathrm{pH}$ effect on the 
(a)

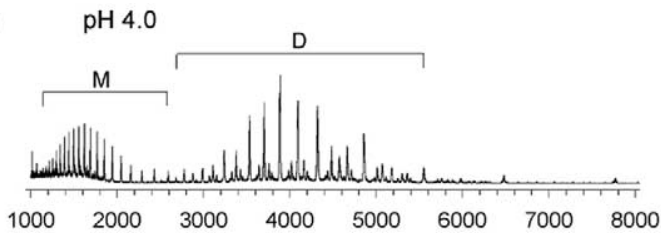

(b)

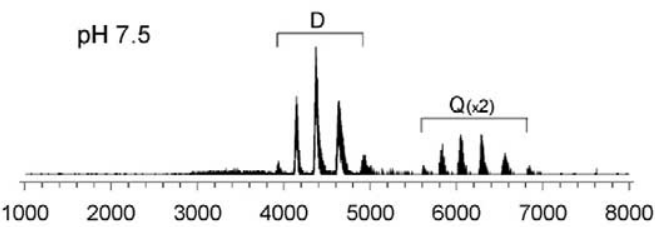

(c)

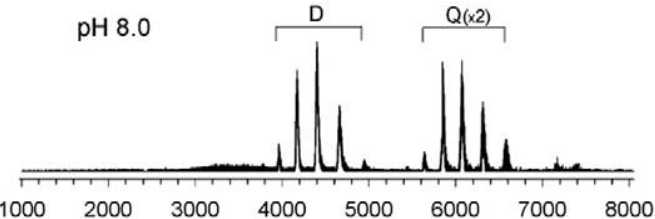

(d)

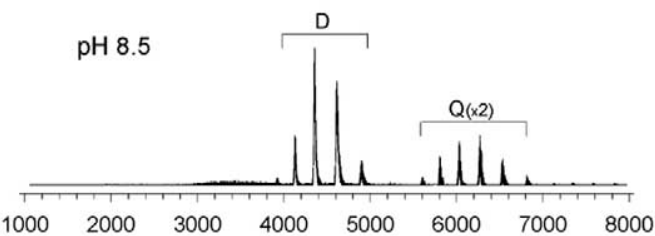

(e)

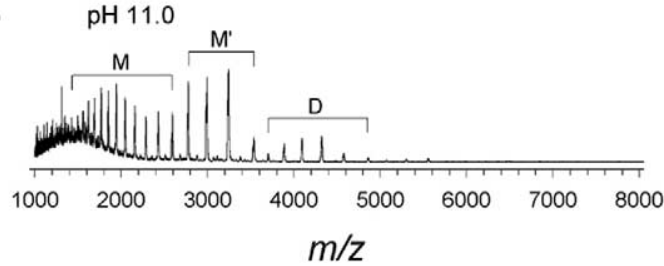

Figure 4. ESI-MS spectra of SaNeuB at (a) $\mathrm{pH}=4.0$; (b) $\mathrm{pH}=7.5$; (c) $\mathrm{pH}=8.0 ;(\mathbf{d}) \mathrm{pH}=8.5 ;(\mathbf{e}) \mathrm{pH}=11.0$. Q: tetramer; $\mathrm{D}$ : dimer; $\mathrm{M}, \mathrm{M}^{\prime}$ : monomer. The accelerating/declustering potential DP and DP2 were set to 100 and $80 \mathrm{~V}$, respectively. The pressure in the collision cell was $7.1 \times 10^{-3}$ torr.

tetrameric abundance in ESI-MS spectra resembles closely with the $\mathrm{pH}$-dependent activity. The tetramer appears only between $\mathrm{pH}$ 6.5-8.5, which shows similar narrow $\mathrm{pH}$ range for optimal activity profile. On the other hand, the dimeric form is relatively insensitive to the $\mathrm{pH}$ variation, predominantly appearing throughout the $\mathrm{pH}$ range of $4.0-11.0$. The results strongly suggest that the tetrameric form is most likely to be the primary species responsible for enzyme activity. Therefore, we demonstrated that ESI-MS spectra can reflect subtle change on protein quaternary structure of SaNeuB at different $\mathrm{pH}$, which correlates well with the enzyme activity.

\section{The Enzyme NeuB is a Tetramer in Solution}

EcNeuB is homologous to $\mathrm{SaNeuB}(70 \%)$ and share $56 \%$ sequence identity at amino acid level. Under identical ESI-MS conditions at $\mathrm{pH}$ 8.0, a similar mass pattern showing major dimer and minor tetramer was observed for $^{\circ} \mathrm{EcNeuB}^{\circ}$ as $^{\circ}$ shown $^{\circ}$ in $^{\circ}$ Figure $^{\circ} 5 .^{\circ} \operatorname{In}^{\circ}$ contrast $^{\circ}$ to ${ }^{\circ}$ the absence of monomer in the study of SaNeuB, monomeric form was observed in the EcNeuB spectrum in small but relatively substantial percentage (20\%) with wide $^{\circ}$ charge ${ }^{\circ}$ state $^{\circ} 24+{ }^{\circ}$ to $^{\circ} 12+^{\circ}\left(\mathrm{m} / \mathrm{z} 1600^{\circ} \sim{ }^{\circ} 3200\right) .{ }^{\circ}$ The monomer of EcNeuB may arise from the dissociation in the electrospray ionization process, which further implies that the quaternary structure of EcNeuB is more fragile than SaNeuB. The comparison between SaNeuB and $\mathrm{EcNeuB}$ shows that they have similar quaternary structures, whereas the latter has better stability than the former.

Interestingly, a single peak of $135 \mathrm{kDa}$, which is between the masses of trimer and tetramer, was observed $^{\circ}$ in $^{\circ}$ the $^{\circ}$ size-exclusion $^{\circ}$ chromatography $^{\circ}[27]^{\circ} .^{\circ} \mathrm{A}$ contradictory result was obtained by a similar experiment with deduced native molecular mass of NeuB as $78^{\circ} \mathrm{kDa}^{\circ}\left[26{ }^{\circ} .^{\circ}\right.$ Nevertheless, ${ }^{\circ}$ in $^{\circ}$ our $^{\circ}$ native ${ }^{\circ} \mathrm{PAGE}^{\circ}$ study, the NeuB appeared as a single species with mass of approximately $140 \mathrm{kDa}$. The detection of the tetrameric $\mathrm{NeuB}$ is supported by the additional evidence from analytical ultracentrifugation experiments that both Sa$\mathrm{NeuB}$ and EcNeuB showed a major peak of molecular weight $160 \mathrm{kDa}$ with similar sedimentation coefficients (unpublished data). More importantly, the structure specificity of tetramer has been demonstrated on the disrupted tetrameric assembly by shift to extreme high or low $\mathrm{pH}$ in ultracentrifugation experiment, in accord with our ESI-MS result. In summary, though these

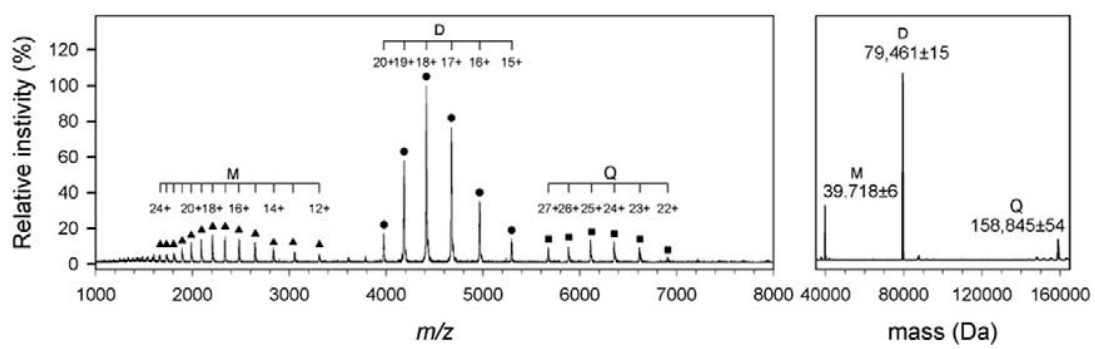

Figure 5. ESI-MS raw spectrum of native sialic acid synthase from E. coli under nondenaturing conditions (a) and the deconvoluted mass spectrum (b). Q: tetramer; D: dimer; M: monomer. The accelerating/declustering potential DP and DP2 were set to 100 and $80 \mathrm{~V}$, respectively. The pressure in the collision cell was $7.1 \times 10^{-3}$ torr. 


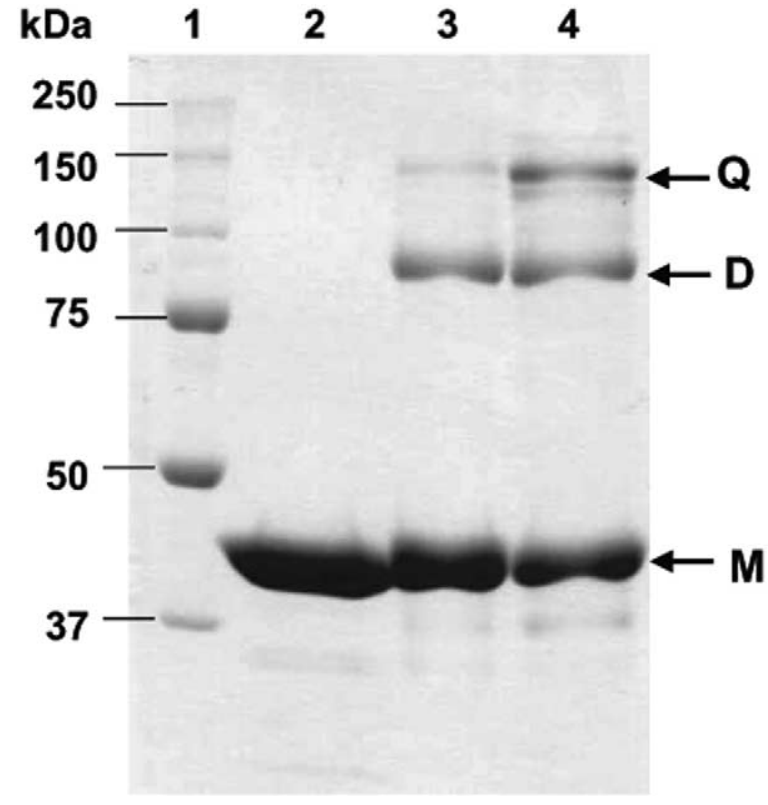

Figure 6. SDS-PAGE of subunit cross-linking of SaNeuB with DMA and DMS. Lane 1, Mark 12 wide range protein standards (Novel Experimental Technology, San Diego, USA); lane 2, unmodified SaNeuB; lane3: reaction with DMA (8.6 ̊); lane 4, reaction with DMS $(11 \AA)$. The arrows show the positions of cross-linked dimer (D) and tetramer (Q) at approximately 80 and $160 \mathrm{kDa}$ from top to bottom, respectively.

classical techniques displayed inconsistent mass assignment of the oligomeric states, yet a common conclusion can be drawn on the existence of a single multimeric form of NeuB in solution.

In addition to providing measurement of structural integrity of NeuB similar to the above-mentioned methods, the ESI-MS pattern can offer additional information $^{\circ}$ on $^{\circ}$ subunit ${ }^{\circ}$ interaction $s^{\circ}[16] .^{\circ}$ In $^{\circ}$ this $^{\circ}$ study,${ }^{\circ}$ it ${ }^{\circ}$ is worth noting that changes in instrumental parameters induced variation of tetramer/dimer ratio. Hence, we speculate that the tetramer observed in the ESI-MS study presents the native structure whereas the dimer may arise from dissociation of the tetramer. It is interesting to further study if the observed dimeric species can $^{\circ}$ reflect $^{\circ}$ the $^{\circ}$ geometry $^{\circ}$ of $^{\circ}$ tetrameric ${ }^{\circ}$ association ${ }^{\circ}$ of NeuB. The statement can be supported by comparison with ${ }^{\circ}$ other ${ }^{\circ}$ related ${ }^{\circ}$ enzymes ${ }^{\circ}$ shown ${ }^{\circ}$ as ${ }^{\circ}$ follow.

The reaction of NeuB is analogous to those catalyzed by 3-deoxy-D-manno-octalosonate 8-phosphate (KDO8P) synthase and 3-deoxy-D-arabino-heptlosonate (DAHP7P) synthase. The KDO8P synthase and DAHP7P synthase are PEP-dependant as NeuB and the structures have been resolved ${ }^{\circ}$ by $^{\circ} \mathrm{X}$-ray ${ }^{\circ}$ crystallography $^{\circ}[35-38] .{ }^{\circ}$ Particularly, they are also mechanistically and structurally relevant. Although there is no obvious sequence homology among KDO8P synthase, DAHP7P synthase and NeuB, it is coincident that the former two structurally resolved proteins are homotetramers in which each subunit consists of an $\alpha / \beta$-barrel. In addition to the same tetrameric structure, the KDO8P synthase and the DAHP7P synthase consist of two tight dimers in the X-ray structure. The connection is further strengthened by the similarity in the distribution of structural elements including $\alpha$-helix and $\beta$-strand. The secondary structure analysis revealed that EcNeuB (32.9\% $\alpha$-helix and $15.7 \% \beta$-strand) is similar to KDO8P synthase (35.2\% $\alpha$-helix and $19.4 \% \beta$-strand) and DAHP7P synthsase (38.0\% $\alpha$-helix and $16.0 \% \beta$-strand) [27]. By 9 tuning ${ }^{\circ}$ the ${ }^{\circ}$ declustering potential ${ }^{\circ}$ and $^{\circ}$ collision ${ }^{\circ}$ cell pressure, ${ }^{\circ}$ the ${ }^{\circ} \mathrm{ESI}-\mathrm{MS}{ }^{\circ}$ pattern ${ }^{\circ}{ }^{\circ}{ }^{\circ} \mathrm{KDO} 8 \mathrm{P}^{\circ}$ also ${ }^{\circ}$ shows ${ }^{\circ}$ transformation from tetramer to exclusively dimer (unpublished data), similar to the observed SaNeuB. Whether the tetrameric ${ }^{\mathrm{Neu}}{ }^{\circ}{ }^{\circ}$ compasses $^{\circ}{ }^{\circ}$ dimeric $^{\circ}$ dimer $^{\circ}{ }^{\circ} \mathrm{KDO} 8 \mathrm{P}$, leading to the bimodal distribution of dimer and tetramer in ESI-MS, presents an interesting question. The hypothesis prompted us to perform the following cross-linking experiment.

\section{Proposed Model of Tetramer NeuB as Dimeric Dimers}

SaNeuB was subjected to the subunit cross-linking experiment by using bifunctional linkers of different lengths including dimethyl adipimidate (DMA, $8.6 \AA$ ) and dimethyl $1^{\circ}$ suberimidate $\left(\mathrm{DMS},{ }^{\circ} 11^{\circ} \AA\right)^{\circ}[39]^{\circ}{ }^{\circ} \mathrm{As}^{\circ}$ shown $^{\circ}$ in Figure ${ }^{\circ} 6,{ }^{\circ}$ dimer $^{\circ}$ was $^{\circ}$ observed $^{\circ}$ as $^{\circ}$ the ${ }^{\circ}$ major ${ }^{\circ}$ cross-linked species in company with trace amount of the tetramer when DMA was utilized. In contrast, the usage of longer linker DMS led to more significant appearance of the tetrameric form. Based on the cross-linking experiment in which only dimeric and tetrameric structures were produced in SDS-PAGE, it is geometrically possible to propose the ${ }^{9}$ tetrameric ${ }^{9} \operatorname{model}^{\circ} \mathrm{as}^{\circ} \mathrm{a}^{\circ} \mathrm{dimer}^{\circ}$ of $^{\circ} \operatorname{dimers}^{\circ}$ (Figure 7 ). The model suggests the existence of two distinct symmetric interfaces between monomer-monomer and dimerdimer in tetrameric SaNeuB.

When the noncovalently-bound protein assembly is transferred from solution phase to gaseous state, some interaction elements are affected to different extent and subsequently fail to preserve the quaternary structure. In general, electrostatic interaction is greatly enhanced in the absence of solvent, and hence stabilizes complexesqound by ${ }^{\circ}$ electrostatic forces [12]. The hydrophobic interaction, on the contrary, seems to be weakened

(A) DMA $(8.6 \AA)$
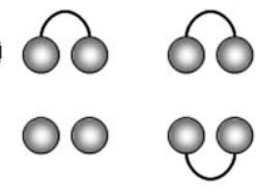

(B) DMS (11.0 Â)
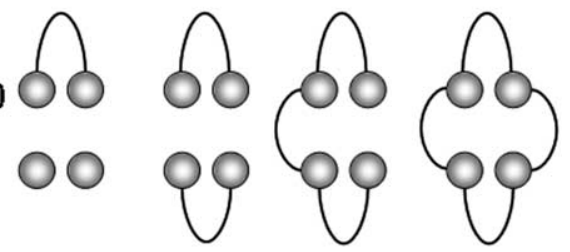

Figure 7. Proposed model of tetrameric $\mathrm{SaNeuB}$ as dimeric dimers. Subunit cross-linking with DMA (8.6 ̊) produced mainly dimer as the cross-linked product, while the reaction with DMS (11 $\AA$ ) cross-linker gave dimer and tetramer. 
since almost all the solvents are stripped off during the desolvation process. It is possible that hydrophobic interaction may exist in the dimer-dimer interface which can easily collapse.

If the hydrophobic interaction is a determining factor for the dimer-dimer interface, the chance to observe the tetrameric NeuB as the major species is highly compromised. The presence of water or salts in multimeric protein complexes has been found to be critical for maintaining ${ }^{\circ}$ the $e^{\circ}$ intact $^{\circ}$ complex $^{\circ}$ in $^{\circ}$ the $^{\circ}$ gas $^{\circ}$ phase $^{\circ}$ [17]. These molecules appear to be fairly typical of noncovalent protein assembly and substantial peak broadening on the peak width of higher oligomeric species is usually ${ }^{\circ}$ bserved ${ }^{\circ}$ ' mass $^{\circ}$ spectra 916$]$. Because the peak width (FWHM) are approximately both 20 Da for dimer and ${ }^{\circ}$ tetramer ${ }^{\circ}$ (Figure ${ }^{\circ}$ ), , neither ${ }^{\circ}$ significant ${ }^{\circ}$ difference $^{\circ}$ in peak'broadening ${ }^{9}{ }^{9}{ }^{9}$ mass increase from the association of water molecules can be found between dimer and tetramer. This phenomenon is different from most measurements ${ }^{\circ}$ on $^{\circ}$ noncovalent ${ }^{\circ}$ assemblies, ${ }^{\circ}$ where ${ }^{\circ}$ broad peak widths are always present in higher multimeric forms presumably due to the inclusion of water or salts $\left[31,{ }^{\circ} 40\right] .{ }^{\circ} \mathrm{As}^{\circ}$ Shown $^{\circ}$ in $^{\circ}$ Figure $^{\circ} 1$, $^{\circ}$ the $e^{\circ}$ inclusion ${ }^{\circ}$ of ${ }^{\circ}$ the ${ }^{\circ}$ Tris molecules significantly increases the intensity of the tetramer, which strongly implies the important roles of salts or water in tetrameric integrity. Tris buffer was also found to be the optimal condition for SaNeuB activity. Therefore, deficiency of enough number of water or salts in the tetramer during ESI process may account for its observed low abundance in ESI-MS spectra. Though HEPES shows similar enhancement in the activity assay (unpublished data), it severely interferes with ESI-MS measurement. The structures of KDO8P synthase and DAHP7P synthase imply that $\mathrm{NeuB}$ is an asymmetric tetramer composed of two dimers with close contacts within dimers but weaker interactions between dimers, leading to the dominant dimer in the mass spectra. The KDO8P synthase also undergoes similar tetramer/dimer dissociation pattern in the mass spectra in different instrument parameters (unpublished data).

\section{Conclusions}

The present study constitutes the first ESI-MS investigation of quaternary organization of sialic acid synthase as a tetramer. High declustering/accelerating potential and sufficient collisional cooling are essential for the preservation of tetramer. The incomplete preservation of the tetrameric sialic acid synthase can be well explained by the gas-phase dissociation of the tetramer due to excess internal energy that is unable to disperse in the gas phase. The close resemblance of the $\mathrm{pH}$ dependent appearance of tetramer in ESI-MS to the activity profile revealed its significance for catalysis.

Together with the cross-linking experiment, a model of dimeric dimers was proposed for the quaternary assembly of NeuB. The study by Jurchen and Williams provides convincing evidence that the gas-phase com- plex can clearly retain a memory of the solution where it forms, and the solution-phase conformation of these ions may play a role in the dissociation pattern in the gas $^{\circ}$ phase $^{\circ}[13]^{\circ}{ }^{\circ}$ When ${ }^{\circ}$ the ${ }^{\circ}$ ions $^{\circ}$ are $^{\circ}$ activated ${ }^{\circ}$ by ${ }^{\circ}$ many low-energy collisions, the dissociation is largely symmetric. Thus, we expected that the gas-phase dissociation of the tetrameric NeuB due to insufficient internal energy relaxation can correlate to the solution-phase structure, and further mirror the NeuB architecture as dimeric dimers in solution.

\section{Acknowledgments}

The authors thank Professor Joseph A. Loo, Department of Biochemistry and Biological Chemistry, University of Los Angeles, for helpful suggestion and comments on the work. This work was supported ${ }^{\circ}$ by $^{\circ}$ the ${ }^{\circ}$ National $^{\circ}$ Science ${ }^{\circ}$ Council $^{\circ}$ (NSC-91-2113-M-001050 and NSC-92-2113-M-001-065) and Academia Sinica (Taipei, Taiwan). The authors also thank the High-Throughput Facility for Proteomic Research for the Q-TOF mass spectrometer.

\section{References}

1. Feild, M. J.; Nguyen, D. C.; Armstrong, F. B. Amino Acid Sequence of Salmonella typhimurium Branched-Chain Amino Acid Aminotransferase. Biochemistry 1989, 28, 5306-5310.

2. Jones, S.; Thornton, J. M. Principles of Protein-Protein Interactions. Proc. Natl. Acad. Sci. U.S.A. 1996, 93, 13-20.

3. van den Heuvel, R. H.; Heck, A. J. Native Protein Mass Spectrometry: From Intact Oligomers to Functional Machineries. Curr. Opin. Chem. Biol. 2004, 8, 519-526.

4. Lengqvist, J.; Svensson, R.; Evergren, E.; Morgenstern, R.; Griffiths, W. J. Observation of an Intact Noncovalent Homotrimer of Detergent-Solubilized Rat Microsomal Glutathione Transferase-1 by Electrospray Mass Spectrometry. J. Biol. Chem. 2004, 279, 13311-13316.

5. McCammon, M. G.; Hernandez, H.; Sobott, F.; Robinson, C. V. Tandem Mass Spectrometry Defines the Stoichiometry and Quaternary Structural Arrangement of Tryptophan Molecules in the Multiprotein Complex TRAP. J. Am. Chem. Soc. 2004, 126, 5950-5951.

6. Halgand, F.; Wessel, P. M.; Laprevote, O.; Dumas, R. Biochemical and Mass Spectrometric Evidence for Quaternary Structure Modifications of Plant Threonine Deaminase Induced by Isoleucine. Biochemistry 2002, 41, 13767-13773.

7. Bernstein, S. L.; Liu, D.; Wyttenbach, T.; Bowers, M. T.; Lee, J. C.; Gray, H. B.; Winkler, J. R. $\alpha$-Synuclein: Stable Compact and Extended Monomeric Structures and pH Dependence of Dimer Formation. J. Am. Soc. Mass Spectrom. 2004, 15, 14351443.

8. Chen, X. L.; Qu, L. B.; Zhang, T.; Liu, H. X.; Yu, F.; Yu, Y.; Liao, X.; Zhao, Y. F. The Nature of Phosphorylated Chrysin-Protein Interactions Involved in Noncovalent Complex Formation by Electrospray Ionization Mass Spectroscopy. Anal. Chem. 2004, 76, 211-217.

9. Hanson, C. L.; Videler, H.; Santos, C.; Ballesta, J. P.; Robinson, C. V. Mass Spectrometry of Ribosomes from Saccharomyces cerevisiae: Implications for Assembly of the Stalk Complex. J. Biol. Chem. 2004, 279, 42750-42757.

10. Winston, R. L.; Fitzgerald, M. C. Mass Spectrometry as a Readout of Protein Structure and Function. Mass Spectrom. Rev. 1997, 16, 165-179.

11. Aquilina, J. A.; Benesch, J. L.; Bateman, O. A.; Slingsby, C.; Robinson, C. V. Polydispersity of a Mammalian Chaperone: Mass Spectrometry Reveals the Population of Oligomers in 
$\alpha$ B-crystallin. Proc. Natl. Acad. Sci. U.S.A. 2003, 100, 10611-10616.

12. Loo, J. A. Electrospray Ionization Mass Spectrometry: A Technology for Studying Noncovalent Macromolecular Complexes. Int. J. Mass Spectrom. 2000, 200, 175-186.

13. Jurchen, J. C.; Williams, E. R. Origin of Asymmetric Charge Partitioning in the Dissociation of Gas-Phase Protein Homodimers. J. Am. Chem. Soc. 2003, 125, 2817-2826.

14. Loo, J. A. Studying Noncovalent Protein Complexes by Electrospray Ionization Mass Spectrometry. Mass Spectrom. Rev. 1997, 16, 1-23.

15. Rogniaux, H.; Sanglier, S.; Strupat, K.; Azza, S.; Roitel, O.; Ball, V.; Tritsch, D.; Branlant, G.; Van Dorsselaer, A. Mass Spectrometry as a Novel Approach to Probe Cooperativity in Multimeric Enzymatic Systems. Anal. Biochem. 2001, 291, $48-61$.

16. Nettleton, E. J.; Sunde, M.; Lai, Z.; Kelly, J. W.; Dobson, C. M. Robinson, C. V. Protein Subunit Interactions and Structural Integrity of Amyloidogenic Transthyretins: Evidence from Electrospray Mass Spectrometry. J. Mol. Biol. 1998, 281, 553564.

17. Rostom, A. A.; Sunde, M.; Richardson, S. J.; Schreiber, G.; Jarvis, S.; Bateman, R.; Dobson, C. M.; Robinson, C. V. Dissection of Multi-Protein Complexes Using Mass Spectrometry: Subunit Interactions in Transthyretin and Retinol-Binding Protein Complexes. Proteins 1998, Suppl. 2, 3-11.

18. Rogniaux, H.; Van Dorsselaer, A.; Barth, P.; Biellmann, J. F.; Barbanton, J.; van Zandt, M.; Chevrier, B.; Howard, E.; Mitschler, A.; Potier, N.; Urzhumtseva, L.; Moras, D.; Podjarny, A. Binding of Aldose Reductase Inhibitors: Correlation of Crystallographic and Mass Spectrometric Studies. J. Am. Soc. Mass Spectrom. 1999, 10, 635-647.

19. Fandrich, M.; Tito, M. A.; Leroux, M. R.; Rostom, A. A.; Hartl, F. U.; Dobson, C. M.; Robinson, C. V. Observation of the Noncovalent Assembly and Disassembly Pathways of the Chaperone Complex MtGimC by Mass Spectrometry. Proc. Natl. Acad. Sci. U.S.A. 2000, 97, 14151-14155.

20. Pinkse, M. W.; Maier, C. S.; Kim, J. I.; Oh, B. H.; Heck, A. J. Macromolecular Assembly of Helicobacter Pylori Urease Investigated by Mass Spectrometry. J. Mass Spectrom. 2003, 38, 315-320.

21. Light-Wahl, K. J.; Schwartz, B. L.; Smith, R. D. Observation of the Noncovalent Quaternary Associations of Proteins by Electrospray Ionization Mass Spectrometry. J. Am. Chem. Soc. 1994, 116, 5271-5278.

22. Sanglier, S.; Ramstrom, H.; Haiech, J.; Leize, E.; Dorsselaer, A. V. Electrospray Ionization Mass Spectrometry Analysis Revealed a $310 \mathrm{kDa}$ Noncovalent Hexamer of HPr kinase/ phosphatase from Bacillus subtilis. Int. J. Mass Spectrom. 2002, 219, 681-696.

23. Rubens, C. E.; Wessels, M. R.; Heggen, L. M.; Kasper, D. L. Transposon Mutagenesis of Type III Group B Streptococcus: Correlation of Capsule Expression with Virulence. Proc. Natl. Acad. Sci. U.S.A. 1987, 84, 7208-7212.

24. Chaffin, D. O.; Beres, S. B.; Yim, H. H.; Rubens, C. E. The Serotype of Type Ia and III Group B Streptococci is Determined by the Polymerase Gene within the Polycistronic Capsule Operon. J. Bacteriol. 2000, 182, 4466-4477.

25. Annunziato, P. W.; Wright, L. F.; Vann, W. F.; Silver, R. P. Nucleotide Sequence and Genetic Analysis of the neuD and
neuB Genes in Region 2 of the Polysialic Acid Gene Cluster of Escherichia coli K1. J. Bacteriol. 1995, 177, 312-319.

26. Suryanti, V.; Nelson, A.; Berry, A. Cloning, Over-Expression, Purification, and Characterization of N-Acetylneuraminate Synthase from Streptococcus agalactiae. Protein Expr. Purif. 2003, 27, 346-356.

27. Hwang, T.-S.; Hung, C.-H.; Teo, C.-F.; Chen, G.-T.; Chang, L.-S.; Chen, S.-F.; Chen, Y.-J.; Lin, C.-H. Structural Characterization of Escherichia coli Sialic Acid Synthase. Biochem. Biophys. Res. Commun. 2002, 295, 167-173.

28. Warren, L. The Thiobarbituric Acid Assay of Sialic Acids J. Biol. Chem. 1959, 234, 1971-1975.

29. Davies, G. E.; Stark, G. R. Use of Dimethyl Suberimidate, a Cross-Linking Reagent, in Studying the Subunit Structure of Oligomeric Proteins. Proc. Natl. Acad. Sci. U.S.A. 1970, 66, 651656.

30. Chiou, S. H.; Chang, W. P.; Lin, H. K. A Re-evaluation of the Molecular Size of Duck $\varepsilon$-Crystallin and Its Comparison with Avian Lactate Dehydrogenases. Biochim. Biophys. Acta 1988,957,313-317.

31. Sanglier, S.; Leize, E.; Van Dorsselaer, A.; Zal, F. Comparative ESI-MS Study of Approximately 2.2 MDa Native Hemocyanins from Deep Sea and Shore Crabs: From Orotein Oligomeric State to Biotope. J. Am. Soc. Mass Spectrom. 2003, 14, 419-429.

32. Schmidt, A.; Bahr, U.; Karas, M. Influence of Pressure in the First pumping Stage on Analyte Desolvation and Fragmentation in Nano-ESI MS. Anal. Chem. 2001, 73, 6040-6046.

33. Tahallah, N.; Pinkse, M.; Maier, C. S.; Heck, A. J. The Effect of the Source Pressure on the Abundance of Ions of Noncovalent Protein Assemblies in an Electrospray Ionization Orthogonal Time-of-Flight Instrument. Rapid Commun. Mass Spectrom. 2001, 15, 596-601.

34. Krutchinsky, A. N.; Chernushevich, I. V.; Spicer, V. L.; Ens, W.; Standing, K. G. Collisional Damping Interface for an Electrospray Ionization Time-of-Flight Mass Spectrometer. J. Am. Chem. Soc. Mass Spectrom. 1998, 9, 569-579.

35. Radaev, S.; Dastidar, P.; Patel, M.; Woodard, R. W.; Gatti, D. L. Structure and Mechanism of 3-Deoxy-D-Manno-Octulosonate 8-Phosphate Synthase. J. Biol. Chem. 2000, 275, 9476-9484.

36. Shumilin, I. A.; Kretsinger, R. H.; Bauerle, R. H. Crystal Structure of Phenylalanine-Regulated 3-Deoxy-D-Arabino-Heptulosonate7-Phosphate Synthase from Escherichia coli. Struct. Fold Des. 1999, 7, 865-875.

37. Wagner, T.; Shumilin, I. A.; Bauerle, R.; Kretsinger, R. H. Structure of 3-Deoxy-D-Arabino-Heptulosonate-7-Phosphate Synthase from Escherichia coli: Comparison of the $\mathrm{Mn}(2+)^{* 2-}$ Phosphoglycolate and the $\mathrm{Pb}(2+)^{*} 2$-Phosphoenolpyruvate Complexes and Implications for Catalysis. J. Mol. Biol. 2000, 301, 389-399.

38. Izard, T.; Lawrence, M. C.; Malby, R. L.; Lilley, G. G.; Colman, P. M. The Three-Dimensional Structure of N-Acetylneuraminate Lyase from Escherichia coli. Structure 1994, 2, 361-369.

39. Green, N. S.; Reisler, E.; Houk, K. N. Quantitative Evaluation of the Lengths of Homobifunctional Protein Cross-Linking Reagents Used as Molecular Rulers. Prot. Sci. 2001, 10, 12931304.

40. Rostom, A. A.; Robinson, C. V. Disassembly of Intact Multiprotein Complexes in the Gas Phase. Curr. Opin. Struct. Biol. 1999, 9, 135-141. 